\title{
Physical Layer Security for Cooperative NOMA Systems
}

\author{
Jianchao Chen, Liang Yang, and Mohamed-Slim Alouini, Fellow, IEEE
}

\begin{abstract}
In this correspondence, we investigate the physical layer security for cooperative non-orthogonal multiple access (NOMA) systems, where both amplify-and-forward (AF) and decode-and-forward (DF) protocols are considered. More specifically, some analytical expressions are derived for secrecy outage probability (SOP) and strictly positive secrecy capacity (SPSC). Results show that AF and DF almost achieve the same secrecy performance. Moreover, asymptotic results demonstrate that the SOP tends to a constant at high signal-to-noise ratio (SNR). Finally, our results show that the secrecy performance of considered NOMA systems is independent of the channel conditions between the relay and the poor user.
\end{abstract}

Index Terms - Non-orthogonal multiple access (NOMA), relaying, secrecy outage probability (SOP), strictly positive secrecy capacity (SPSC).

\section{INTRODUCTION}

As one of the promising key techniques of fifth generation (5G) wireless networks, non-orthogonal multiple access (NOMA) has been applied in various areas. NOMA utilizes the power domain to achieve multiple-access strategies, which is unlike the conventional orthogonal multiple access structures, for instance, frequency division multiple access. Since the NOMA technique exploits the new dimension of the power domain [1], it has the potential to be integrated with existing MA paradigms. On the other hand, the technique of cooperative transmission can form a virtual multiple-input multipleoutput (MIMO) scheme to process data cooperatively, which can enhance the communication reliability for the users who are in poor channel conditions. Recently, applying cooperative transmission to NOMA has been considered in [2], [3]. A hybrid decode-and-forward (DF) and amplify-and-forward (AF) relaying strategy was studied in [4] for a cooperative NOMA system with multiple relays. In [5], the outage performance of a DF relaying NOMA system with two-stage relay selection (TSRS) method was analyzed. The results in [5] show that the proposed TSRS outperforms the conventional max-min relay

Copyright (c) 2015 IEEE. Personal use of this material is permitted. However, permission to use this material for any other purposes must be obtained from the IEEE by sending a request to pubs-permissions@ieee.org.

Manuscript received Nov 1, 2017; revised Dec 26, 2017; accepted Dec 29, 2017. This work was in part supported by the National Natural Science Foundation of China (NSFC) under Grant 61372096 and 61671160, the Department of Education of Guangdong Province (No. 2016KZDXM050), and the open research fund of National Mobile Communications Research Laboratory, Southeast University (No. 2018D01). The review of this paper was coordinated by Dr Richard Souza.

J. Chen and L. Yang are with the Department of Communication Engineering, Guangdong University of Technology, Guangzhou, China.

M.-S. Alouini is with the Computer, Electrical, and Mathematical Science and Engineering Division, King Abdullah University of Science and Technology, Thuwal, Saudi Arabia. selection criteria. More recently, the authors in [6] studied a cooperative NOMA scenario with the help of an AF relay and derived an approximate outage probability expression.

Due to the broadcast nature of the wireless communication, the concept of physical layer security was initially proposed by Wyner in [7]. Physical layer security, as an appealing method to implement secure communication, has received considerable interest recently. Until now, the secrecy performance analysis for different systems has been extensively considered in the literature, such as MIMO (transmit antenna selection and maximal ratio combining) [8], cooperative diversity [9], energy harvesting [10], and cognitive radio [11]. However, these works are not related to the NOMA system. Recently, the physical layer security for 5G NOMA in stochastic geometry networks was considered in [12]. Later, an extension of [12] was presented in [13] where two different structures were proposed to improve the secrecy performance for singleantenna and multiple-antenna stochastic geometry networks, respectively. In [14], a new design of NOMA under secrecy considerations was proposed and the optimal designs of decoding order, transmission rates, and power allocated to each user were investigated. To the best of the authors' knowledge, there are few works related to the analysis of the physical layer security in cooperative NOMA systems. Thus, this is the main motivation of this work.

In this correspondence, we consider a cooperative NOMA system with a single relay where both AF and DF protocols are considered. More specifically, we assume that the NOMA users are affected by an eavesdropper. With this setting, analytical expressions for the secrecy outage probability (SOP) and strictly positive secrecy capacity (SPSC) are derived. At high signal-to-noise ratio (SNR) regimes, results show that the secrecy performance of AF and DF NOMA systems is the same. Finally, it is observed that the secrecy performance of NOMA systems at high SNR is independent of the channel condition between the relay and the poor user.

\section{System And Channel Models}

Consider a NOMA system including a base station (BS), two users (UE1, poor user, and UE2, strong user), a relay, and an eavesdropper (E) in a cellular network, where BS is located in the center of the cell, and users and $\mathrm{E}$ are very close to the cell bound. Therefore, we assume that there are no direct links between BS and the users, as well as E. We further assume that all nodes in the network have single antennas and all the channels suffer from independent Rayleigh fading. Similar to the most assumptions in the literature, we assume that $\mathrm{E}$ 
can obtain the messages from relay to users, including the forwarding protocol at relays, and decoding method at users.

In the first time slot, the superimposed mixture, $\left(\alpha_{1} s_{1}+\right.$ $\left.\alpha_{2} s_{2}\right)$, are broadcast from BS to the relay, where $s_{i}(i=1,2)$ is the unit power signal received by user $\mathrm{i}$ and $\alpha_{i}$ is the power allocation coefficient. To fulfill UE1's QoS requirements, we set that $\alpha_{1} \geq \alpha_{2}$ and define the power allocation coefficients satisfy the equation $\alpha_{1}^{2}+\alpha_{2}^{2}=1$ [15]. Then, the received signal at the relay can be written as

$$
y_{r}=h_{r}\left(\alpha_{1} s_{1}+\alpha_{2} s_{2}\right) \sqrt{P_{s}}+\omega_{r},
$$

where $h_{r}$ denotes the channel gain between the BS and the relay and $\omega_{r}$ is the additive white Gaussian noise with zero mean and variance $N_{0}$. In the next, we describe the transmission procedures for AF and DF protocols, respectively.

\section{A. Amplify-and-Forward}

For $\mathrm{AF}$, in the second time slot, the relay amplifies and forwards its received signals to the two users. Then, the received signal at the user $i$ is

$$
y_{r, i}^{A F}=\beta y_{r} g_{r, i}+\omega_{r, i}
$$

where $\beta$ is the amplifying factor given by $\beta=\sqrt{\frac{P_{r}}{P_{s}\left|h_{r}\right|^{2}+N_{0}}}$ [16], $g_{r, i}$ is the channel between relay and UE $i, \omega_{r, i}$ denotes the additive Gaussian noise with zero mean and variance $N_{0}$, and $P_{r}$ is the transmit power of the relay. We assume that $P_{r}=P_{s}$, then $\beta=\sqrt{\frac{1}{\left|h_{r}\right|^{2}+\frac{1}{\rho}}}$, where $\rho=P_{s} / N_{0}=P_{r} / N_{0}$ denotes the average SNR of the legal links. In (2), $g_{r, i}$ is the small-scale fading coefficient between the relay and user $i$.

Meanwhile, due to the broadcast nature of wireless transmissions, E also receives a copy of the signal from the relay and then the received signal at $\mathrm{E}$ is given by

$$
y_{r, E}^{A F}=\beta y_{r} g_{r, E}+\omega_{r, E},
$$

where $g_{r, E}$ denotes the channel gain from the relay to $\mathrm{E}$ and $\omega_{r, E}$ is the additive Gaussian noise with variance $N_{E}$. Similar to [1][13], successive interference cancellation (SIC) can be applied in the NOMA system to decode the signal $s_{i}$ of user $i$. Hence, the AF-based transmission capacity from the relay to user $i$ can be written as

$$
C_{r, i}^{A F}=0.5 \log _{2}\left(1+\gamma_{r, i}^{A F}\right),
$$

where $\gamma_{r, 1}^{A F}=\frac{\left|g_{r, 1}\right|^{2}\left|h_{r}\right|^{2} \alpha_{1}^{2}}{\left|g_{r, 1}\right|^{2}\left|h_{r}\right|^{2} \alpha_{2}^{2}+\frac{1}{\rho}\left|g_{r, 1}\right|^{2}+\frac{1}{\rho}\left|h_{r}\right|^{2}+\frac{1}{\rho^{2}}}$ denotes the instantaneous signal-to-interference-plus-noise ratio (SINR) at UE1 and $\gamma_{r, 2}^{A F}=\frac{\rho\left|h_{r}\right|^{2}\left|g_{r, 2}\right|^{2} \alpha_{2}^{2}}{\left|g_{r, 2}\right|^{2}+\left|h_{r}\right|^{2}+\frac{1}{\rho}}$ is the received SNR at UE2.

In this work, like [12][13], we consider the worst case and assume $\mathrm{E}$ has the multiuser detection ability. In particular, parallel interference cancellation (PIC) is used at E to distinguish the superimposed mixture. Then, the channel capacity from the relay to E can be expressed as

$$
C_{E, i}^{A F}=0.5 \log _{2}\left(1+\gamma_{E, i}^{A F}\right),
$$

where $\gamma_{E, i}^{A F}=\frac{\rho \rho_{E}\left|h_{r}\right|^{2}\left|g_{r, E}\right|^{2} \alpha_{i}^{2}}{\rho_{E}\left|g_{r, E}\right|^{2}+\rho\left|h_{r}\right|^{2}+1}$ is the received SNR at E and $\rho_{E}=P_{r} / N_{E}$ is the average SNR of the illegal link between the relay and $\mathrm{E}$.
With (4) and (5), the secrecy rate of the AF-based NOMA systems for user $i$ is given by

$$
C_{i}^{A F}=\left\lceil C_{r, i}^{A F}-C_{E, i}^{A F}\right\rceil^{+},
$$

where $\lceil x\rceil^{+}=\max \{x, 0\}$.

\section{B. Decode-and-Forward}

For DF case, the relay first decodes its received superimposed message from BS and then re-encodes and forwards it to the destination. Then, the received signals at UE $i$ and $E$ can be shown to be given by

$$
y_{r, \Theta}^{D F}=g_{r, \Theta}\left(\alpha_{1} s_{1}+\alpha_{2} s_{2}\right) \sqrt{P_{s}}+\omega_{r, \Theta},
$$

where $\Theta \in i, E$. The channel capacity of a DF relaying system is $\min \left\{C_{B S-R}, C_{R-U E i}\right\}$, where $C_{B S-R}$ and $C_{R-U E i}$ denote the capacity from $\mathrm{BS}$ to relay and relay to $\mathrm{UE} i$, respectively. Hence, the capacity of the main channels is

$$
C_{B S-U E}^{D F, i}=\frac{1}{2} \log _{2}\left(1+\min \left\{\frac{\rho\left|h_{r}\right|^{2} \alpha_{1}^{2}}{\rho\left|h_{r}\right|^{2} \alpha_{2}^{2}+1}, \frac{\rho\left|g_{r, 1}\right|^{2} \alpha_{1}^{2}}{\rho\left|g_{r, 1}\right|^{2} \alpha_{2}^{2}+1}\right\}\right)
$$

and

$$
C_{B S-U E}^{D F, i}=0.5 \log _{2}\left(1+\min \left\{\rho\left|h_{r}\right|^{2} \alpha_{2}^{2}, \rho\left|g_{r, 2}\right|^{2} \alpha_{2}^{2}\right\}\right),
$$

Similarly, the capacity of the eavesdropping channel is

$$
C_{R-E}^{D F, i}=0.5 \log _{2}\left(1+\rho_{E}\left|g_{r, E}\right|^{2} \alpha_{i}^{2}\right) .
$$

Finally, the secrecy capacity of the DF-based NOMA systems for UE $i$ can be expressed as

$$
C_{i}^{D F}=\left\lceil C_{B S-U E}^{D F, i}-C_{R-E}^{D F, i}\right\rceil^{+} .
$$

\section{Secrecy Performance Analysis}

In this section, we evaluate the secrecy performance in terms of SOP and SPSC metrics. To obtain more insights, we also provide the asymptotic SOP analysis.

A. Secrecy Performance for AF-based NOMA Systems

1). SOP Analysis

In NOMA systems, two signals are transmitted from the source to UE1 and UE2 with the help of a relay, respectively. Therefore, outage happens when either $C_{1}^{A F}$ or $C_{2}^{A F}$ falls below their own target rates. With this definition, the SOP can be expressed as

$$
\begin{aligned}
& \mathrm{SOP}^{A F}=\operatorname{Pr}\left(C_{1}^{A F}<R_{1} \text { or } C_{2}^{A F}<R_{2}\right) \\
& =1-\operatorname{Pr}\left(\frac{1+\gamma_{r, 1}^{A F}}{1+\gamma_{E, 1}^{A F}}>C_{t h}^{1}, \frac{1+\gamma_{r, 2}^{A F}}{1+\gamma_{E, 2}^{A F}}>C_{t h}^{2}\right)=1-\operatorname{Pr} 1,
\end{aligned}
$$

where $R_{i}$ is the target data rate for user $i$ and $C_{t h}^{i}=2^{2 R_{i}}$. From (12), we can observe that the variables $\gamma_{r, 1}^{A F}, \gamma_{r, 2}^{A F}, \gamma_{E, 1}^{A F}$ and $\gamma_{E, 2}^{A F}$ are correlated, which makes untractable exact analysis. Hence, we focus on the analysis for high SNR regime and adopt the following upper bounds $\gamma_{r, 1}^{A F}<\frac{\alpha_{1}^{2}}{\alpha_{2}^{2}}, \gamma_{r, 2}^{A F}<$ $\frac{\rho \rho\left|h_{r}\right|^{2}\left|g_{r, 2}\right|^{2} \alpha_{2}^{2}}{\rho\left|g_{r, 2}\right|^{2}+\rho\left|h_{r}\right|^{2}}$, and $\gamma_{E, i}^{A F}<\frac{\rho \rho_{E}\left|h_{r}\right|^{2}\left|g_{r, E}\right|^{2} \alpha_{i}^{2}}{\rho_{E}\left|g_{r, E}\right|^{2}+\rho\left|h_{r}\right|^{2}}$. Then, an upper bound of Pr1 can be obtained as

$$
\begin{aligned}
\operatorname{Pr} 1< & \operatorname{Pr}\left(\frac{\rho \rho_{E}\left|h_{r}\right|^{2}\left|g_{r, E}\right|^{2}}{\rho_{E}\left|g_{r, E}\right|^{2}+\rho\left|h_{r}\right|^{2}}<\xi_{1}, \frac{\rho \rho\left|h_{r}\right|^{2}\left|g_{r, 2}\right|^{2} \alpha_{2}^{2}}{\rho\left|g_{r, 2}\right|^{2}+\rho\left|h_{r}\right|^{2}}\right. \\
& \left.>\frac{C_{t h}^{2} \rho \rho_{E}\left|h_{r}\right|^{2}\left|g_{r, E}\right|^{2} \alpha_{2}^{2}}{\rho_{E}\left|g_{r, E}\right|^{2}+\rho\left|h_{r}\right|^{2}}+C_{t h}^{2}-1\right)
\end{aligned}
$$


where $\xi_{1}=\frac{1-\alpha_{2}^{2} C_{t h}^{1}}{\alpha_{1}^{2} \alpha_{2}^{2} C_{t h}^{1}}$ and $\alpha_{1}^{2}>\alpha_{2}^{2}\left(C_{t h}^{1}-1\right)$ due to $\xi_{1}=$ $\frac{\alpha_{1}^{2}-\alpha_{2}^{2}\left(C_{t h}^{1}-1\right)}{\alpha_{1}^{2} \alpha_{2}^{2} C_{t h}^{1}}>0$. Otherwise, $S O P^{A F}=1$. By using the broad-using inequality $x y /(x+y) \leq \min \{x, y\}$ [17], Pr1 can be further approximated as

$$
\begin{aligned}
& \operatorname{Pr} 1<\operatorname{Pr}\left(\min \left\{\rho\left|h_{r}\right|^{2}, \rho_{E}\left|g_{r, E}\right|^{2}\right\}<\xi_{1}, \min \left\{\left|h_{r}\right|^{2},\left|g_{r, 2}\right|^{2}\right\}\right. \\
& \left.>\min \left\{C_{t h}^{2}\left|h_{r}\right|^{2}, A\left|g_{r, E}\right|^{2}\right\}+B\right) .
\end{aligned}
$$

where $A=\frac{C_{t h}^{2} \rho_{E}}{\rho}$ and $B=\frac{C_{t h}^{2}-1}{\rho \alpha_{2}^{2}}$. However, it is still difficult to obtain a closed-form for (14). To address this, we use the fact $P\left(\varepsilon_{1}, \varepsilon_{2}\right)=P\left(\varepsilon_{1}\right)-P\left(\varepsilon_{1}, \overline{\varepsilon_{2}}\right)$, where $\overline{\varepsilon_{2}}$ denotes the complementary event of $\varepsilon_{2}$. Then, we have

$$
\begin{aligned}
& \varepsilon_{1}=\min \left\{\left|h_{r}\right|^{2},\left|g_{r, 2}\right|^{2}\right\}>\min \left\{C_{t h}^{2}\left|h_{r}\right|^{2}, A\left|g_{r, E}\right|^{2}\right\}+B, \\
& \varepsilon_{2}=\min \left\{\rho\left|h_{r}\right|^{2}, \rho_{E}\left|g_{r, E}\right|^{2}\right\}<\xi_{1}
\end{aligned}
$$

With (15) and (16), (14) can be rewritten as

$$
\begin{aligned}
& \operatorname{Pr}\left(\min \left\{\left|h_{r}\right|^{2},\left|g_{r, 2}\right|^{2}\right\}>\min \left\{C_{t h}^{2}\left|h_{r}\right|^{2}, A\left|g_{r, E}\right|^{2}\right\}+B\right) \\
& -\operatorname{Pr}\left(\min \left\{\rho\left|h_{r}\right|^{2}, \rho_{E}\left|g_{r, E}\right|^{2}\right\}>\xi_{1}, \min \left\{\left|h_{r}\right|^{2},\left|g_{r, 2}\right|^{2}\right\}\right. \\
& \left.>\min \left\{C_{t h}^{2}\left|h_{r}\right|^{2}, A\left|g_{r, E}\right|^{2}\right\}+B\right)=P_{1}-P_{2} .
\end{aligned}
$$

From (17), we can observe that the event $\min \left\{\left|h_{r}\right|^{2},\left|g_{r, 2}\right|^{2}\right\}>\min \left\{C_{t h}^{2}\left|h_{r}\right|^{2}, A\left|g_{r, E}\right|^{2}\right\}+B$ can be written as $\min \left\{\left|h_{r}\right|^{2},\left|g_{r, 2}\right|^{2}\right\}>A\left|g_{r, E}\right|^{2}+B$, where we use the fact that $\left|h_{r}\right|^{2}$ should be smaller than $C_{t h}^{2}\left|h_{r}\right|^{2}+B$. Then, $P_{1}$ is given by

$$
\begin{aligned}
& P_{1}=\operatorname{Pr}\left(\left|h_{r}\right|^{2}>A\left|g_{r, E}\right|^{2}+B,\left|g_{r, 2}\right|^{2}>A\left|g_{r, E}\right|^{2}+B\right) \\
& =\int_{0}^{\infty} \operatorname{Pr}\left(\left|h_{r}\right|^{2}>A x+B,\left|g_{r, 2}\right|^{2}>A x+B\right) f_{\left|g_{r, E}\right|^{2}}(x) d x \\
& =\int_{0}^{\infty} e^{-\left(\lambda_{0}+\lambda_{2}\right)(A x+B)} \lambda_{E} e^{-\lambda_{E} x} d x=\frac{\lambda_{E} e^{-\left(\lambda_{0}+\lambda_{2}\right) B}}{\lambda_{0} A+\lambda_{2} A+\lambda_{E}},
\end{aligned}
$$

In (18), $\lambda_{0}, \lambda_{2}$ and $\lambda_{E}$ are the Rayleigh channel parameters corresponding to $\left|h_{r}\right|^{2},\left|g_{r, 2}\right|^{2}$, and $\left|g_{r, E}\right|^{2}$, respectively. On the other hand, $P_{2}$ can be obtained as

$$
\begin{aligned}
P_{2}= & \operatorname{Pr}\left(\min \left\{\rho\left|h_{r}\right|^{2}, \rho_{E}\left|g_{r, E}\right|^{2}\right\}>\xi_{1}, \min \left\{\left|h_{r}\right|^{2},\left|g_{r, 2}\right|^{2}\right\}\right. \\
& \left.>\min \left\{C_{t h}^{2}\left|h_{r}\right|^{2}, A\left|g_{r, E}\right|^{2}\right\}+B\right) \\
= & \operatorname{Pr}\left(\left|h_{r}\right|^{2}>\frac{\xi_{1}}{\rho},\left|g_{r, E}\right|^{2}>\frac{\xi_{1}}{\rho_{E}},\left|h_{r}\right|^{2}>A\left|g_{r, E}\right|^{2}+B,\left|g_{r, 2}\right|^{2}\right. \\
& \left.>A\left|g_{r, E}\right|^{2}+B\right) \\
= & \int_{\frac{\xi_{1}}{\rho_{E}}}^{\infty} e^{-\left(\lambda_{0}+\lambda_{2}\right)(A x+B)} \lambda_{E} e^{-\lambda_{E} x} d x \\
= & \frac{\lambda_{E} e^{-\left(\lambda_{0}+\lambda_{2}\right) B}}{\lambda_{0} A+\lambda_{2} A+\lambda_{E}} \exp \left[-\left(\lambda_{0} A+\lambda_{2} A+\lambda_{E}\right) \frac{\xi_{1}}{\rho_{E}}\right]
\end{aligned}
$$

Finally, with (12), (17), (18), and (19), the SOP for the AF relaying NOMA systems is

$$
\begin{aligned}
& \mathrm{SOP}^{A F}=1-P_{1}+P_{2}=1-\frac{\lambda_{E} e^{-\left(\lambda_{0}+\lambda_{2}\right) B}}{\lambda_{0} A+\lambda_{2} A+\lambda_{E}} \\
& +\frac{\lambda_{E} e^{-\left(\lambda_{0}+\lambda_{2}\right) B}}{\lambda_{0} A+\lambda_{2} A+\lambda_{E}} \exp \left[-\left(\lambda_{0} A+\lambda_{2} A+\lambda_{E}\right) \frac{\xi_{1}}{\rho_{E}}\right] .
\end{aligned}
$$

2). SPSC Analysis
SPSC, one of the fundamental benchmark for secrecy performance, denotes the probability of existence of secrecy capacity [18]. Thus, the SPSC for an AF relaying NOMA system is readily given by

$$
\begin{aligned}
& S P S C^{A F}=\operatorname{Pr}\left(C_{1}^{A F}>0, C_{2}^{A F}>0\right) \\
& =\operatorname{Pr}\left(\gamma_{r, 1}^{A F}>\gamma_{E, 1}^{A F}, \gamma_{r, 2}^{A F}>\gamma_{E, 2}^{A F}\right) \\
& \approx \operatorname{Pr}\left(\frac{\alpha_{1}^{2}}{\alpha_{2}^{2}}>\min \left\{\rho\left|h_{r}\right|^{2}, \rho_{E}\left|g_{r, E}\right|^{2}\right\} \alpha_{1}^{2}, \min \left\{\rho\left|h_{r}\right|^{2}, \rho\left|g_{r, 2}\right|^{2}\right\}\right. \\
& \left.>\min \left\{\rho\left|h_{r}\right|^{2}, \rho_{E}\left|g_{r, E}\right|^{2}\right\}\right) .
\end{aligned}
$$

Using a similar analysis developed in subsection 1), we obtain SPSC as

$$
\begin{aligned}
& S P S C^{A F}=\operatorname{Pr}\left(\min \left\{\rho\left|h_{r}\right|^{2}, \rho\left|g_{r, 2}\right|^{2}\right\}>\rho_{E}\left|g_{r, E}\right|^{2}\right) \\
& -\operatorname{Pr}\left(\left|g_{r, E}\right|^{2}>\frac{1}{\rho_{E} \alpha_{2}^{2}},\left|h_{r}\right|^{2}>\frac{\rho_{E}\left|g_{r, E}\right|^{2}}{\rho},\left|g_{r, 2}\right|^{2}>\frac{\rho_{E}\left|g_{r, E}\right|^{2}}{\rho}\right) \\
& =\int_{0}^{\infty} e^{-\frac{\left(\lambda_{0}+\lambda_{2}\right) \rho_{E}}{\rho} x} \lambda_{E} e^{-\lambda_{E} x} d x \\
& -\int_{\frac{1}{\rho_{E} \alpha_{2}^{2}}}^{\infty} e^{-\frac{\left(\lambda_{0}+\lambda_{2}\right) \rho_{E}}{\rho} x} \lambda_{E} e^{-\lambda_{E} x} d x \\
& =\frac{\lambda_{E}\left[1-\exp \left(-\frac{\lambda_{0}}{\rho \alpha_{2}^{2}}-\frac{\lambda_{2}}{\rho \alpha_{2}^{2}}-\frac{\lambda_{E}}{\rho_{E} \alpha_{2}^{2}}\right)\right]}{\frac{\lambda_{0} \rho_{E}}{\rho}+\frac{\lambda_{2} \rho_{E}}{\rho}+\lambda_{E}},
\end{aligned}
$$

\section{B. Secrecy Performance for DF-based NOMA Systems}

\section{1). SOP Analysis}

From (8), (9), (10), and (11), the SOP for the DF-based NOMA systems is given by

$$
\begin{aligned}
& \mathrm{SOP}^{D F}=\operatorname{Pr}\left(C_{1}^{D F}<R_{1} \text { or } C_{2}^{D F}<R_{2}\right) \\
& =1-\operatorname{Pr}\left(C_{1}^{D F}>R_{1}, C_{2}^{D F}>R_{2}\right)=1-\operatorname{Pr} 2 .
\end{aligned}
$$

Similar to the analysis done in subsection 1) of $A$, we can obtain an upper bound of instantaneous SINR as $\frac{\rho\left|h_{r}\right|^{2} \alpha_{1}^{2}}{\rho\left|h_{r}\right|^{2} \alpha_{2}^{2}+1} \approx \frac{\rho\left|g_{r, 1}\right|^{2} \alpha_{1}^{2}}{\rho\left|g_{r, 1}\right|^{2} \alpha_{2}^{2}+1} \approx \frac{\alpha_{1}^{2}}{\alpha_{2}^{2}}$. Then, we have $C_{B S-R-U E}^{D F, 1} \approx \frac{1}{2} \log \left(1+\frac{\alpha_{1}^{2}}{\alpha_{2}^{2}}\right)$. Therefore, Pr2 can be expressed as

$\operatorname{Pr} 2<\operatorname{Pr}\left(\left|g_{r, E}\right|^{2}<\frac{\xi_{1}}{\rho_{E}}, \min \left\{\left|h_{r}\right|^{2},\left|g_{r, 2}\right|^{2}\right\}>A\left|g_{r, E}\right|^{2}+B\right)$

$=\operatorname{Pr}\left(\left|g_{r, E}\right|^{2}<\frac{\xi_{1}}{\rho_{E}},\left|h_{r}\right|^{2}>A\left|g_{r, E}\right|^{2}+B,\left|g_{r, 2}\right|^{2}>A\left|g_{r, E}\right|^{2}+B\right)$

$=\int_{\frac{\xi_{1}}{\rho_{E}}}^{\infty} e^{-\lambda_{0}(A x+B)} e^{-\lambda_{2}(A x+B)} \lambda_{E} e^{-\lambda_{E} x} d x$

$=\frac{\lambda_{E} e^{-\left(\lambda_{0}+\lambda_{2}\right) B}}{\lambda_{0} A+\lambda_{2} A+\lambda_{E}}\left\{1-\exp \left[-\left(\lambda_{0} A+\lambda_{2} A+\lambda_{E}\right) \frac{\xi_{1}}{\rho_{E}}\right]\right\}$

Finally, from (23) and (24), the SOP for the DF-based NOMA systems is

$$
\begin{aligned}
& \mathrm{SOP}^{D F}=1-\frac{\lambda_{E} e^{-\left(\lambda_{0}+\lambda_{2}\right) B}}{\lambda_{0} A+\lambda_{2} A+\lambda_{E}} \\
& +\frac{\lambda_{E} e^{-\left(\lambda_{0}+\lambda_{2}\right) B}}{\lambda_{0} A+\lambda_{2} A+\lambda_{E}} \exp \left[-\left(\lambda_{0} A+\lambda_{2} A+\lambda_{E}\right) \frac{\xi_{1}}{\rho_{E}}\right] .
\end{aligned}
$$


Remark 1: Comparing (20) with (25), we can observe that the SOP expressions of AF and DF NOMA systems are the same at high SNR regime.

2). SPSC Analysis

Similar to subsection 1) of $B$, we have $\frac{\rho\left|h_{r}\right|^{2} \alpha_{1}^{2}}{\rho\left|h_{r}\right|^{2} \alpha_{2}^{2}+1} \approx$ $\frac{\rho\left|g_{r, 1}\right|^{2} \alpha_{1}^{2}}{\rho\left|g_{r, 1}\right|^{2} \alpha_{2}^{2}+1} \approx \frac{\alpha_{1}^{2}}{\alpha_{2}^{2}}$. Then, the SPSC for a DF case can be expressed as

$$
\begin{aligned}
& S P S C^{D F}=\operatorname{Pr}\left(C_{1}^{D F}>0, C_{2}^{D F}>0\right) \\
& \approx \operatorname{Pr}\left(\rho_{E}\left|g_{r, E}\right|^{2} \alpha_{2}^{2}<1, \min \left\{\left|h_{r}\right|^{2},\left|g_{r, 2}\right|^{2}\right\}>\frac{\rho_{E}}{\rho}\left|g_{r, E}\right|^{2}\right) \\
& =\operatorname{Pr}\left(\left|g_{r, E}\right|^{2}<\frac{1}{\rho_{E} \alpha_{2}^{2}},\left|h_{r}\right|^{2}>\frac{\rho_{E}}{\rho}\left|g_{r, E}\right|^{2},\left|g_{r, 2}\right|^{2}>\frac{\rho_{E}}{\rho}\left|g_{r, E}\right|^{2}\right) \\
& =\int_{0}^{\frac{1}{\rho_{E} \alpha_{2}^{2}}} \lambda_{E} \exp \left[-\left(\frac{\lambda_{0} \rho_{E}}{\rho}+\frac{\lambda_{2} \rho_{E}}{\rho}+\lambda_{E}\right) x\right] d x \\
& =\frac{\lambda_{E}\left[1-\exp \left(-\frac{\lambda_{0}}{\rho \alpha_{2}^{2}}-\frac{\lambda_{2}}{\rho \alpha_{2}^{2}}-\frac{\lambda_{E}}{\rho_{E} \alpha_{2}^{2}}\right)\right]}{\frac{\lambda_{0} \rho_{E}}{\rho}+\frac{\lambda_{2} \rho_{E}}{\rho}+\lambda_{E}},
\end{aligned}
$$

From above analysis, we can find that the secrecy performance of an AF NOMA system is very close to that of the DF case, which can be verified by the simulation results in section IV.

Remark 2: From the analytical expressions of SOP and SPSC for both AF and DF protocols, we can see that the secrecy performance at high SNR is independent of the channel between the relay and UE1. Furthermore, we note that the instantaneous SINR at high SNR tends to a constant, i.e. $\frac{\alpha_{1}^{2}}{\alpha_{2}^{2}}$. According to the definition of the cooperative NOMA, the poor user should be quickly connected with a low data rate, while the strong one should be served opportunistically [15]. Therefore, for high $\rho$, the poor user compared to the strong user is easy to obtain a secure communication, which in turn results in the conclusion that the secrecy performance of NOMA systems is mostly determined by $\left|h_{r}\right|^{2},\left|g_{r, 2}\right|^{2}$ and $\left|g_{r, E}\right|^{2}$ at high SNR regimes.

\section{Asymptotic SOP Analysis}

To investigate the asymptotic secrecy performance, we also provide an asymptotic SOP analysis.

From (12), $\gamma_{E, 1}^{A F} \approx \rho_{E}\left|g_{r, E}\right|^{2} \alpha_{1}^{2}$ and $\gamma_{E, 2}^{A F}$ become a constant when $\gamma_{r, 2}^{A F} \rightarrow \infty$. Therefore, the SOP performance of AFbased NOMA systems can be asymptotically expressed as

$$
\mathrm{SOP}^{A F} \approx \operatorname{Pr}\left(\frac{1+\frac{\alpha_{1}^{2}}{\alpha_{2}^{2}}}{1+\rho_{E}\left|g_{r, E}\right|^{2} \alpha_{1}^{2}}<C_{t h}^{1}\right)=\exp \left[-\frac{\lambda_{E} \xi_{1}}{\rho_{E}}\right],
$$

Similarly, from (23), the asymptotic expression for a DF based NOMA is given by

$$
\begin{aligned}
& \operatorname{SOP}^{D F}=\operatorname{Pr}\left(C_{1}^{D F}<R_{1} \text { or } C_{2}^{D F}<R_{2}\right) \\
& \approx \operatorname{Pr}\left(C_{1}^{D F}<R_{1}\right) \approx \operatorname{Pr}\left(\frac{1+\frac{\alpha_{1}^{2}}{\alpha_{2}^{2}}}{1+\rho_{E}\left|g_{r, E}\right|^{2} \alpha_{1}^{2}}<C_{t h}^{1}\right) \\
& =\exp \left[-\frac{\lambda_{E} \xi_{1}}{\rho_{E}}\right]
\end{aligned}
$$

From (27) and (28), we can find that the asymptotic expression of the AF relaying NOMA is equal to that of its corresponding DF case when $\rho \rightarrow \infty$. Moreover, from (12) and (23), for both relaying protocols, we can observe that the capacity of the strong user's link must be larger than its target rate $R_{2}$. Therefore, the SOP expressions of AF and DF relaying NOMA systems are only affected by the channels between the relay and $\mathrm{E}$ when $\rho \rightarrow \infty$.

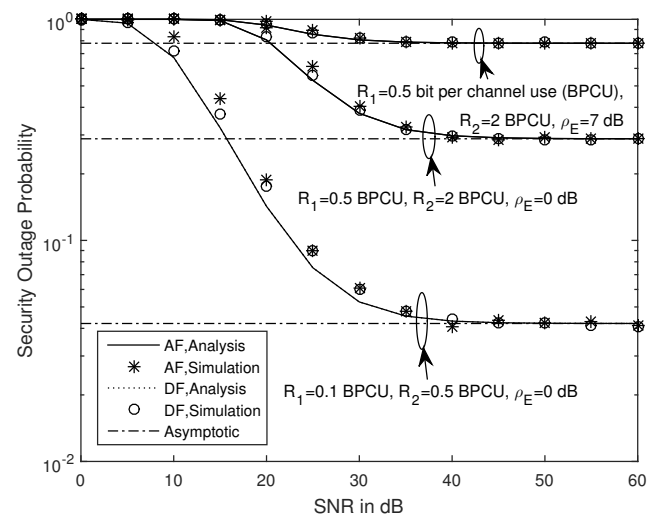

Fig. 1. Secrecy outage probability of the AF and DF relaying NOMA systems. $\lambda_{0}=\lambda_{1}=\lambda_{2}=1$ and $\alpha_{1}=0.86$.

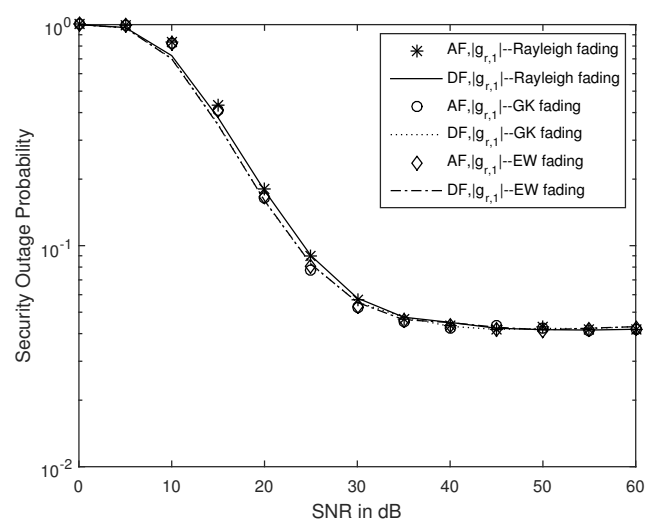

Fig. 2. SOP versus $\rho$ for different fading models of $g_{r, 1} . \alpha_{1}=0.86$, $R_{1}=0.1 \mathrm{BPCU}, R_{2}=0.5 \mathrm{BPCU}$.

\section{Numerical Results}

In this section, we present some insightful numerical results to disclose the secrecy performance of cooperative NOMA systems. Also, Monte-Carlo simulation results are provided to verify our analytical results.

In Fig. 1, we plot the SOP curves for different parameters. From Fig. 1, we can observe that the SOP of both AF and DF relaying NOMA tends to be a constant when $\rho$ is large enough, which is proved in the subsection $C$. For such a case, the SOP performance is only related to $R_{i}$ and $\rho_{E}$. However, to ensure a reliable communication, small values for the target rate and the average SNR of the eavesdropper link should be chosen since the superimposed message is transmitted to two 


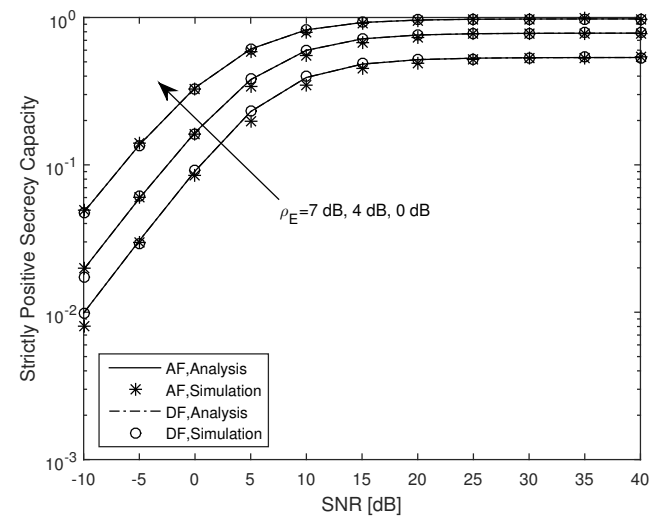

Fig. 3. SPSC for different values of $\rho_{E} \cdot \alpha_{1}=0.86, R_{1}=0.01 \mathrm{BPCU}$, $R_{2}=0.05$ BPCU.

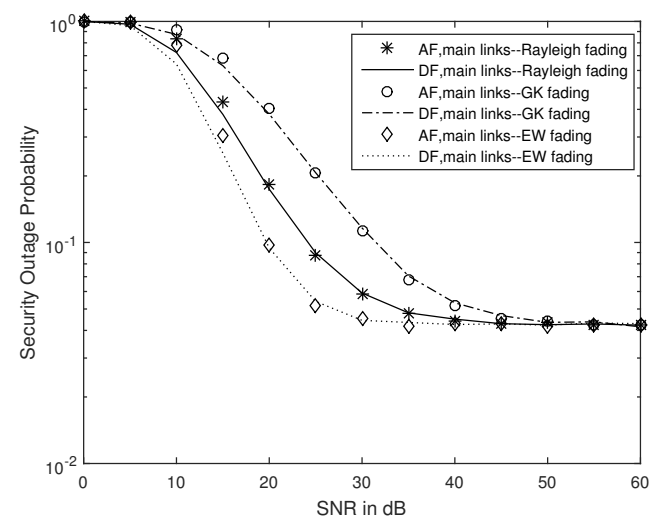

Fig. 4. SOP versus $\rho$ for different fading models of the main channels. $\alpha_{1}=0.86, R_{1}=0.1 \mathrm{BPCU}, R_{2}=0.5 \mathrm{BPCU}$.

different destinations, which means that the NOMA systems have a high secure requirement than the traditional point-topoint communication.

In Fig. 2, the simulation results of SOP versus $\rho$ when $\left|g_{r, 1}\right|^{2}$ experiencing different fading are presented. More specifically, we consider three different channel fading models for $\left|g_{r, 1}\right|^{2}$, i.e., Rayleigh fading, EW fading [19], and GK fading [20]. We can see that the curves corresponding to different fading models are close to each other, which verifies the Remark 2 that the values of $\left|g_{r, 1}\right|^{2}$ do not affect the SOP performance at high SNR regimes.

In Fig. 3, we plot the SPSC curves for both AF and DF relaying NOMA systems versus $\rho$ for different values of $\rho_{E}$. Simulation results verify the accuracy of our derivations. Also, the SPSC of the AF relaying strategy is very close to that of the DF case. Furthermore, similar to the SOP analysis, some same observations can be obtained.

Simulations of SOP versus $\rho$ when the main channels experiencing different fading are plotted in Fig. 4. This figure demonstrates that the curves are close to each other when $\rho \rightarrow \infty$. Thus, we can conclude that the secrecy performance of the cooperative NOMA systems are independent of the main channels.

\section{CONCLUSIONS}

In this correspondence, we analyzed the secrecy performance of cooperative NOMA systems for both AF and DF protocols. Results show that the SOP of NOMA systems tends to a constant at high SNR regimes. Moreover, for high $\rho$, the poor user compared to the strong user is easy to obtain a secure communication, which in turn results in the conclusion that the secrecy performance of NOMA systems is mostly determined by $\left|h_{r}\right|^{2},\left|g_{r, 2}\right|^{2}$ and $\left|g_{r, E}\right|^{2}$.

\section{REFERENCES}

[1] Z. Ding et al., "Application of non-orthogonal multiple access in LTE and 5G networks," IEEE Commun. Mag., vol. 55, no. 2, pp. 185-191, Feb. 2017.

[2] Z. Ding, M. Peng, and H. V. Poor, "Cooperative non-orthogonal multiple access in $5 \mathrm{G}$ systems," IEEE Commun. Lett., vol. 19, no. 8, pp. 14621465, Aug. 2015

[3] Y. Liu, Z. Ding, M. Elkashlan, and H. V. Poor, "Cooperative nonorthogonal multiple access with simultaneous wireless information and power transfer" IEEE J. Sel. Areas Commun., vol. 34, no. 4, pp. 938953, Apr. 2016

[4] Y. Liu, G. Pan, H. Zhang and M. Song, "Hybrid decode-forward and amplify-forward relaying with non-orthogonal multiple access," IEEE Access, vol. 4, pp. 4912-4921, Feb. 2016.

[5] Z. Ding, H. Dai, and H. V. Poor, "Relay selection for cooperative NOMA," IEEE Commun. Lett., vol. 5, no. 4, pp. 416-419, Aug. 2016.

[6] X. Liang, Y. Wu, D. W. K. Ng, Y. Zuo S. Jin, and H. Zhu, "Outage performance for cooperative NOMA transmission with an AF relay," IEEE Commun. Lett., vol. 21, no. 11, pp. 2428-2431, Nov. 2017.

[7] A. D. Wyner, "The wire-tap channel," Bell Syst. Tech. J., vol. 54, no. 8, pp. 1355-1387, 1975 .

[8] N. Yang, H. Suraweera, I. Collings, and C. Yuen, "Physical layer security of TAS/MRC with antenna correlation," IEEE Trans. Inf. Forensics Security, vol. 8, no. 1, pp. 254-259, Jan. 2013

[9] Y. Zou, X. Wang and W. Shen, "Optimal Relay Selection for physicallayer security in cooperative wireless networks," IEEE J. Sel. Areas Commun., vol. 31, no. 10, pp. 2099-2111, Oct. 2013.

[10] M. Zhang and Y. Liu, "Energy harvesting for physical-layer security in OFDMA networks," IEEE Trans. Inf. Forensics Security, vol. 11, no. 1, pp. 154-162, Jan. 2016.

[11] Y. Zou, X. Wang and W. Shen, "Physical-layer security with multiuser scheduling in cognitive radio networks," IEEE Trans. Commun., vol. 61, no. 12, pp. 5103-5113, Dec. 2013.

[12] Z. Qin, Y. Liu, Z. Ding, Y. Gao, and M. Elkashlan, "Physical layer security for $5 \mathrm{G}$ non-orthogonal multiple access in large-scale networks," in Proc. Int. commun. Conf. (ICC), May 2016, PP. 1-6.

[13] Y. Liu, Z. Qin, M. Elkashlan, Y. Gao and L. Hanzo, "Enhancing the physical layer security of non-orthogonal multiple access in large-scale networks," IEEE Trans. Wireless Commun., vol. 16, no. 3, pp. 1656-1672, Mar. 2017.

[14] B. He, A. Liu, N. Yang and V. K. N. Lau, "On the design of secure non-orthogonal multiple access systems," IEEE J. Sel. Areas Commun., vol. 35, no. 10, pp. 2196-2206, Oct. 2017.

[15] Z. Ding, L. Dai, and H. V. Poor, "MIMO-NOMA design for small packet transmission in the internet of things," IEEE Access, vol. 4, pp. 13931405, Aug. 2016.

[16] C. S. Patel, G. L. Stuber, and T. G. Pratt, "Statistical properties of amplify and forward relay fading channels," IEEE Trans. Veh. Technol., vol. 55, no. 1, pp. 1-9, Jan. 2006

[17] M. K. Simon, and M. S. Alouini, Digital Communication Over Fading Channels, 2nd ed. Hoboken, NJ, USA: Wiley, 2005.

[18] X. Liu, "Probability of strictly positive secrecy capacity of the ricianrician fading channel," IEEE Wireless Commun. Lett., vol. 2, no. 1, pp. 50-53, Feb. 2013.

[19] P. Wang et al., "Performance analysis for relay-aided multihop BPPM FSO communication system over exponentiated Weibull fading channels with pointing error impairments," IEEE Photon. J., vol. 7, no. 4, Aug. 2015.

[20] C. Yoon, H. Lee and J. Kang, "Effect of generalized-K fading on the performance of symmetric coordinate interleaved orthogonal designs," IEEE Commun. Lett., vol. 18, no. 4, pp. 588-591, Apr. 2014. 